tions indicate that a parallel to-and-fro raster is more efficient than a zig-zag one for a field uniform to $2.8 \%$ (with a $2.5 \mathrm{~mm}$ spot size) where the raster spacing must be $5 \mathrm{~mm}$ vertically between turning points.

With a parallel to-and-fro raster, typical rise times required in the scanning magnets (specified from zero to the full deflection of $150 \mathrm{~mm}$ ) are $t$ (horizontal) $=45 \mathrm{~ms}$ and $t$ (vertical) $=300 \mathrm{~ms}$.

The peak power required for the driving electronics is $2 \mathrm{~J} / t$, where $J$ is the stored energy of the magnetic field in joules. With careful beam design the magnetic energies can be kept low, say $75 \mathrm{~J}$ for the horizontal deflector and $300 \mathrm{~J}$ for the vertical, so the peak powers $P$ for the driving electronics would be modest: $P$ (horizontal, for 75 joules) $=3.3 \mathrm{KW} ; P$ (vertical, for 300 joules) $=2.0 \mathrm{KW}$.

\section{Scanning in range}

The depth required in tissue is typically 5 to $20 \mathrm{~cm}$, shallower tumours being treated with low energy proton machines. The corresponding particle energies are 80 $180 \mathrm{MeV} /$ nucleon for protons and alpha particles, $150-330 \mathrm{MeV} /$ nucleon for carbon and 170-400 MeV/nucleon for oxygen. There is no problem in varying the extraction energy using a synchrotron. A cyclotron, however, has a fixed extraction energy so here it will be necessary to reduce the energy by passing the beam through a slab of matter (a degrader) of variable thickness. Unfortunately, the degrader has several undesirable effects which must be analyzed, including:

- increase of the beam phase space by multiple scattering;

- increase of the momentum spread by energy straggling;

- fragmentation of incoming particles, giving lighter ions of roughly the same velocity with a longer range in the patient.

The increase in phase space in a degrader depends on its thickness as well as the scattering angle [14], so the ideal material should have high density and low $Z$. The best compromise is diamond (not readily available in the required thickness); the next best are boron carbide and beryllium. For optimum results, the beam must be focussed onto the centre of the degrader at an optimum convergence angle.

With proton treatment, there is no problem of fragmentation, and the degrader can be placed just in front of the patient, so the beam line can be at fixed energy. With other ions, fragmentation inside the body is inevitable. How vital is it to eliminate fragments produced in an external degrader? This question is still debated. If necessary, the degrader could be placed far from the patient, so that most of the fragments will be lost in the subsequent bending magnets; but then the beam design becomes more complicated.

\section{Conclusions}

Interest in cancer treatment by protons and light ions is increasing rapidly. In the next decade, we can expect to see several more proton accelerators installed in hospitals, and at least one centre for treatment with ions.

\section{REFERENCES}

[1] Wambersie A., Proc. EULIMA Workshop on the Potential Value of Light lon Beam Therapy, Nice (1988).

[2] Mandrillon P. et al., Proc. EPAC, Nice (1990) 1473.

[3] Koehler A.M. and Preston W.M., Radiology 104 (1972) 191.

[4] Castro J.P., Chen G.T.Y. and Blakely E.A. (1985) Radiation Res. 104 (1985) 5263.

[5] Kouris K., Spyron N. and Jackson D.F., Imaging with lonizing Radiations (Surrey University Press, UK) 1982.
[6] Raju M.R., Heavy Particle Radiotherapy (Academic Press) 1980.

[7] Larsson B., Ref. [1], p. 139.

[8] Tubiana M., Dutreix J. and Wambersie A., Radiobiologie (1986); Wambersie A., Barendsen G.W. and Breteau N., Eur. J. Rad. Oncol. 5 (1984) 248.

[9] Curtis H.J., Current Topics in Radiation Res. 3 (1987) 139.

[10] Jongen Y. et al., Ref. [2], p. 274; Blosser $\mathrm{H}$. et al., Michigan State University $\mathrm{Cy}$ clotron Laboratory Report, MSUCL-706a

(1991); Pedroni E. et al., Ref. [2], p. 277.

[11] Castro J.R., Third Workshop on Heavy Charged Particles in Biology and Medicine,

GSI Darmstadt Report 155 N0171-4546 (1987) KOI-5.

[12] Mandrillon P. et al., Ref. [2], p. 1790; Lefèvre P. et al., CERN/PS Internal Report (1991).

[13] Kawashi K. et al., Acta Radio. S364 (1983) 81.

[14] Carli C. and Farley F.J.M., Ref. [2], p. 1796.

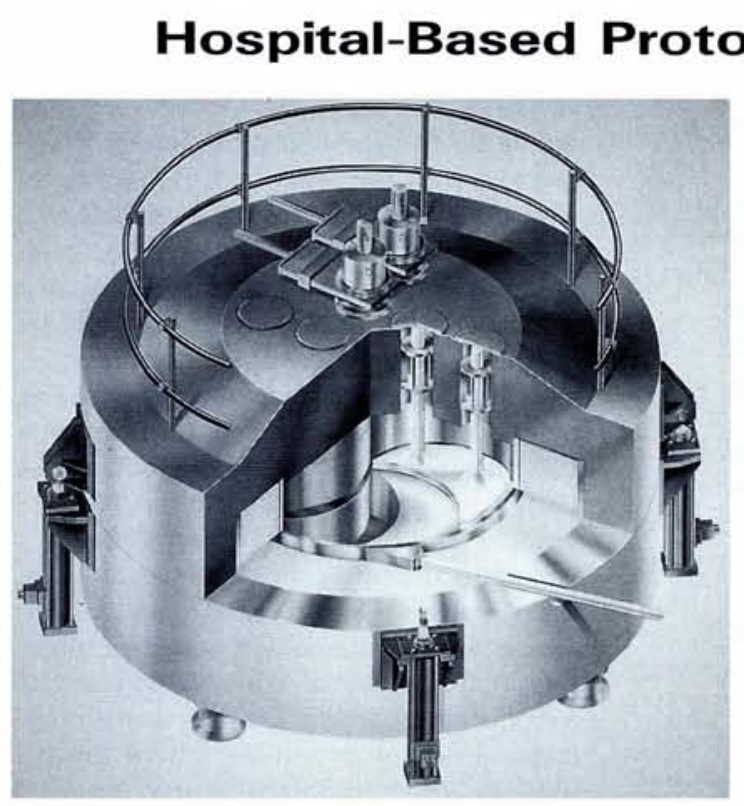

It is generally agreed that among the different kinds of radiation suitable for medical radiotherapy, high energy proton beams exhibit the best ballistic specificity for the selective destruction of cancer cells. Development has been hindered, however, by the size, cost and complexity of the equipment. To overcome these obstacles, a handful of companies are directing research at designing and supplying not only an accelerator, but also turn-key, easily operated, proton therapy facilities for hospitals.

Proton therapy has up to now been carried out at essentially physics-based installations in Zurich, Uppsala, Paris, Nice, Harvard and Berkeley in the USA and Tsukuba and Chiba in Japan. The Commission of the European Community optimistically estimates that some 10 hospital-based facilities are required in Europe over the next 10 years. Longer term interest will be determined by the demonstrated efficacy level of hospital-based proton therapy and whether doctors become convinced scanning systems are foolproof.

\section{IBA Design}

Ion Beam Applications (IBA) is among those companies with the most advanced traction), deep-valley design using $190 \mathrm{~kW}$ in conventional coils. The upper half of the split cyclotron can be quickly raised one metre using hydraulic jacks to allow unrestricted access. This feature, combined with a short pump-down time (30 min), minimizes downtime. To increase the reliability of the system, the number of components is reduced to a minimum.

An unique extraction scheme deflects particles in the hill region so that they pass through a specific "window" at the level of the closed pole edges beyond which the beam encounters a magnetic field gradient at a level below $2.2 \mathrm{~T}$, thus avoiding the use of an expensive electromagnetic tunnel.

The cyclotron would feed 2-4 isocentric gantries of reduced size and cost which allow the the proton beam to scan a patient using both conventional scanning techniques and a novel approach that links the axial sweep magnet field to a dose integrator, guaranteeing uniform dose irrespective of small intensity fluctuations. For conventional step ("voxal") scanning, the new gantry design makes it possible to achieve an infinite source-to-patient distance with a gantry of less than $3 \mathrm{~m}$ in radius. 